\title{
Tube worms promote community change
}

\author{
Ruth Callaway* \\ Biological Sciences, University of Wales Swansea, Singleton Park, Swansea SA4 3SW, UK
}

\begin{abstract}
Dense lawns of tube worms modify their immediate environment. They alter sediment properties, offer refuge from predation and provide a settlement surface for larvae and small organisms. The terebellid polychaete Lanice conchilega is among the tube dwellers that, when present at high densities, alter the community structure of other benthic fauna. However, L. conchilega often occurs individually or at low densities over large areas, and it is not known whether single tubes also affect the surrounding fauna. In this study a low density population of L. conchilega $\left(15.7 \pm 15.6 \mathrm{~m}^{-2}\right)$ was investigated on an exposed beach in South Wales, UK, from May 1998 to April 1999. Effects of single tubes and small groups of 2 to 5 tubes on the benthic community were examined over 1 yr. The relationship between $L$. conchilega and an associated amphipod (Urothoe poseidonis) was studied more closely in the field and the laboratory. Of a total of 56 species, 27 were found exclusively in samples with $L$. conchilega tubes. In comparison with tube-free samples, species richness and abundance of individuals was significantly higher in samples containing L. conchilega tubes. The community structure differed significantly between samples containing groups of tubes and tube-free samples in 10 out of 11 cases and in 9 of 11 cases for samples with 1 tube compared to samples with no tubes. Throughout the year, the polychaete Eumida sanguinea and the haustoriid amphipod U. poseidonis benefited from the presence of L. conchilega. E. sanguinea lives among the fringe filaments of the tube top, and $U$. poseidonis inhabits areas deep in the sediment in close vicinity to the tube. Laboratory experiments indicated that, unlike other haustoriids, the amphipod is not prone to benthopelagic migration but remains in the sediment for long periods of time and may benefit from an improved oxygen supply arising from $L$. conchilega's activity inside the tube. It is concluded that not only groups of tubes, but also single polychaete tubes bioengineer their environment.
\end{abstract}

KEY WORDS: Lanice conchilega · Bioengineering · Habitat engineer · Tube worm · Benthic community $\cdot$ Urothoe poseidonis

Resale or republication not permitted without written consent of the publisher

\section{INTRODUCTION}

Species that actively or passively structure the sea floor and thereby improve or deteriorate environmental conditions for other organisms are referred to as ecosystem engineers (Lawton 1994) or bioengineers (Reise 2002). Examples are manifold. Studies range from maerl beds, kelp forests, colonies of ascidians and Darwin mounds to seagrass meadows, oyster beds and mussel banks (Tsuchiya \& Nishihira 1986, DeGrave 1999, Edgar 1999, Ragnarsson \& Raffaelli 1999, Meyer \& Townsend 2000, Angel \& Ojeda 2001, Castilla et al. 2004, Van Gaever et al. 2004) and have shown that bioengineering affects recruitment, survival and the spatial distribution of benthic macrofauna.
Several tube worm species have been found to possess bioengineering capacities, e.g. Polydora quadrilobata (Khaitov et al. 1999), Loimia sp. and Axionice sp. (Trueblood 1991) and Diopatra cuprea (Ban \& Nelson 1987). Tubes have been found to influence species diversity and abundance (Woodin 1978), the dispersal pattern of other polychaetes (Trueblood 1991), and meiofaunal composition and microbial biomass (Peachy \& Bell 1997, Netto et al. 1999, Phillips \& Lovell 1999). Whilst most effects on diversity or abundance are positive, tube dwellers can negatively affect the survival rate of newly recruited bivalves and polychaetes (Luckenbach 1987, Trueblood 1991).

Most effects of tube worms on other fauna are dependent on their density (Bolam \& Fernandes 2003). 
In this study, the effect of individual tubes and small groups of Lanice conchilega on the benthic fauna was investigated. L. conchilega is one of the most common tube-building polychaetes in temperate sandflats. It is an amphiboreal species found on all coasts of Europe and in both the Atlantic and the Pacific, but is absent from Arctic waters (Holthe 1978). It colonises intertidal and subtidal sediments to depths of about $100 \mathrm{~m}$ and can reach densities of several thousand individuals $\mathrm{m}^{-2}$ (Hertweck 1995, Ropert \& Dauvin 2000). Buhr \& Winter (1977) reported up to 20000 individuals $\mathrm{m}^{-2}$ from shallow subtidal areas off the German coast. The tubes of adult $L$. conchilega have a diameter of ca. $0.5 \mathrm{~cm}$ and are up to $65 \mathrm{~cm}$ long (Ziegelmeier 1952). They consist of sand particles attached to an inner thin organic layer. The particles are composed of a wide range of different materials: silica grains, parts of bivalve and gastropod shells, fragments of sea urchins, foraminiferans and ostracods (Ziegelmeier 1952). The anterior end of the tube protrudes 1 to $4 \mathrm{~cm}$ above the sediment surface and is crowned with a fringe. L. conchilega can switch between deposit and suspension feeding (Buhr 1976), and is preyed upon by wading birds (Petersen \& Exo 1999) and fish (Kühl 1963).

Past studies of intertidal and subtidal areas have shown that dense aggregations of Lanice conchilega comprising several thousand individuals $\mathrm{m}^{-2}$ host a different and often particularly diverse benthic community compared to tube-free areas (Zühlke 2001, Rees et al. 2005). However, L. conchilega does not necessarily occur at high densities, and their tubes are commonly scattered over large areas. Especially in more exposed locations L. conchilega does not reach high densities, probably because the physical force of waves and currents prevents the development of dense lawns or reefs. It has so far not been determined whether the presence of $L$. conchilega affects other members of the macrofauna under such conditions. The present study therefore addressed the following questions: (1) Do single tubes or small groups of $L$. conchilega tubes affect the species richness, abundance and composition of other benthic fauna? (2) What is the nature of the relationships between $L$. conchilega and species benefiting from the presence of the tube worm?

\section{MATERIALS AND METHODS}

The Lanice conchilega population studied was situated in Rhossili Bay, a sandy beach at the western tip of the Gower Peninsula in South Wales, UK (Fig. 1). The study was carried out between May 1998 and April 1999. Rhossili Bay is exposed to waves and strong tidal currents with a tidal range of up to $10 \mathrm{~m}$ (National Tidal and Sea Level Facility [NTSLF], available at www.pol.ac.uk.ntslf; measured at the tide gauge of Mumbles lifeboat station; Fig. 1). The L. conchilega population covered an intertidal area of approximately $0.01 \mathrm{~km}$ around $51^{\circ} 34.08^{\prime} \mathrm{N}, 4^{\circ} 17.88^{\prime} \mathrm{W}$. It was emersed at spring low tides only. The density (mean \pm SD) of adult $L$. conchilega ranged between $1.8 \pm 2.8 \mathrm{~m}^{-2}$ in January 1999 and $34.4 \pm 18.8 \mathrm{~m}^{-2}$ in March 1999 (yearly average $15.7 \pm 15.6 \mathrm{~m}^{-2}$ ). According to the index of dispersion (i.e. variance-to-mean ratio) adult L. Conchilega were randomly distributed, based on numbers $100 \mathrm{~cm}^{-2}$ ( $\mathrm{n}=100$ ) calculated for April, May and June 1998. Tubes were either solitary or in groups of 2 to 5 closely spaced individuals (Fig. 2).

Lanice conchilega communities. To investigate the effects of Lanice conchilega tubes on the other benthic fauna, replicate core samples $\left(32 \mathrm{~cm}^{2}\right.$ surface area, $10 \mathrm{~cm}$ deep) were taken and divided into 3 categories: (1) samples with 2 to 5 closely spaced L. conchilega tubes (= groups of L. conchilega); (2) samples with single tubes; (3) samples with no tubes. Samples were taken monthly from May 1998 to April 1999, except for September 1998 due to logistical problems.

At each sampling, 8 replicate sam-

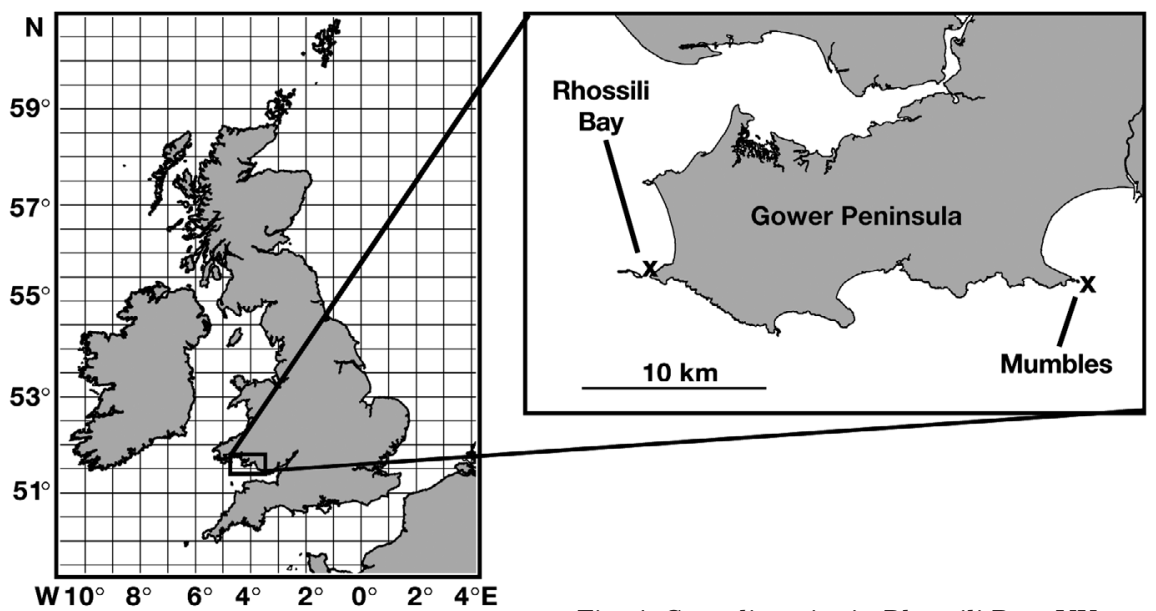

Fig. 1. Sampling site in Rhossili Bay, UK ples were haphazardly taken for all 3 categories. However, upon return to the laboratory it sometimes emerged that the number of $L$. conchilega tubes had been misclassified in the field and samples had to be reallocated to a different category. This was particularly a problem in May and June 1998, when juvenile L. Conchilega were attached to tubes of adults (Callaway 2003a). Uneven numbers of replicates in the different categories (Table 1) were taken into account in the data analyses (see ‘Data analyses' below). 


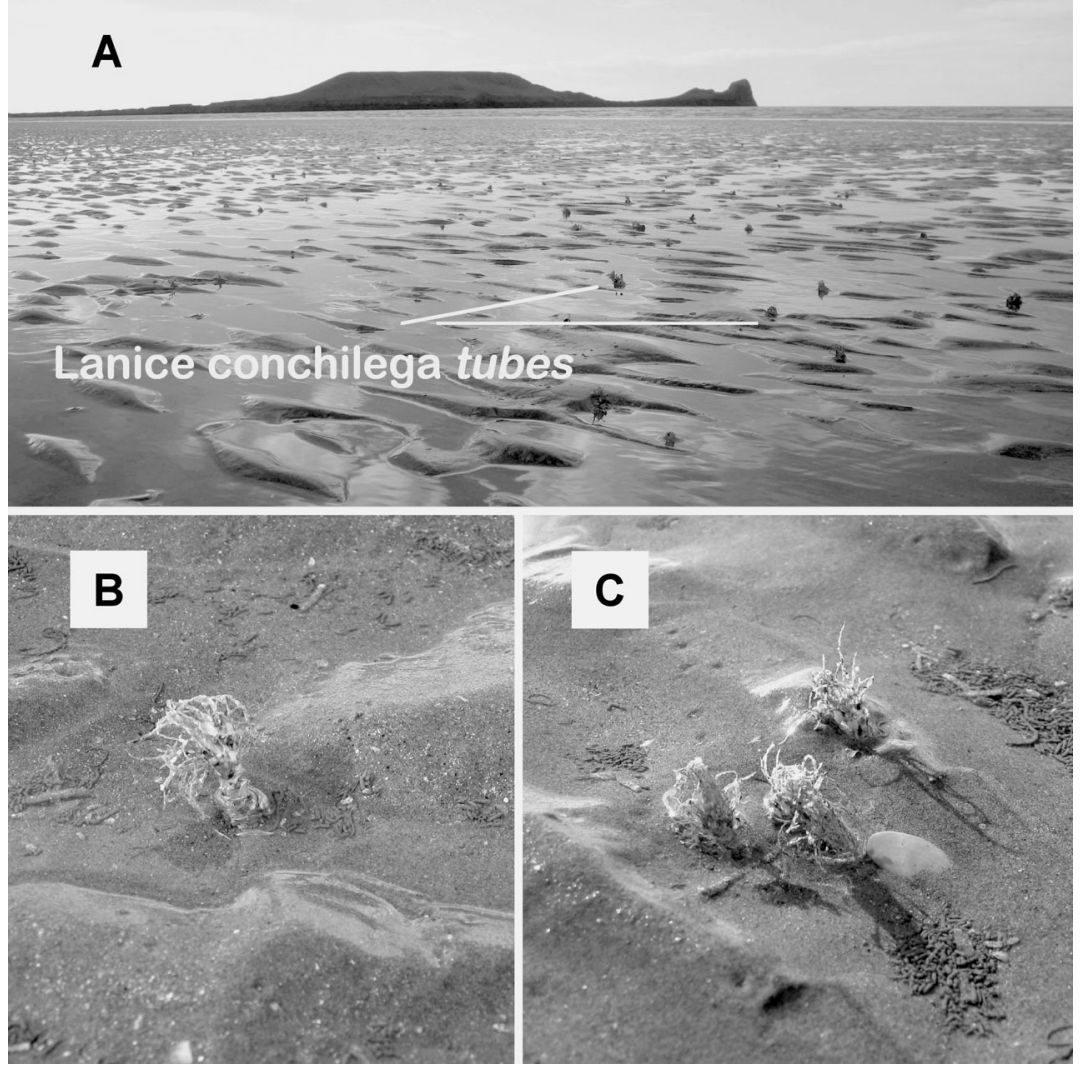

Fig. 2. Lanice conchilega. (A) Tubes in Rhossili Bay, UK; (B) single tube-top; (C) group of tube-tops

Samples were washed through a $0.5 \mathrm{~mm}$ mesh sieve and were either processed immediately or preserved in $70 \%$ ethanol for later processing. All macrofaunal organisms were identified to species level.

Distribution of Urothoe poseidonis around tubes. The amphipod Urothoe poseidonis was commonly associated with Lanice conchilega tubes and studies were carried out to clarify its relationship with the polychaete. The spatial position of $U$. poseidonis in relation to L. conchilega tubes was approximated by analysing its vertical and horizontal position in the sediment. The vertical distribution of $U$. poseidonis was investigated by slicing core sediment samples $\left(32 \mathrm{~cm}^{2}\right)$ with single L. conchilega tubes into $2 \mathrm{~cm}$ sections. Samples were taken in May $1998(\mathrm{n}=12)$ and July $1998(\mathrm{n}=8)$. Each slice was washed through a $0.5 \mathrm{~mm}$ mesh sieve and the macrobenthic fauna identified to species level.

In order to determine the distance of Urothoe poseidonis around Lanice conchilega tubes, samples were taken with different sized corers, with single L. conchilega tubes in the centre of the core samples. The corers had diameters of 1.8, 3.2 and $5.4 \mathrm{~cm}$ and were nested like Russian dolls inside each other (March and April 1998, n = 22). The L. conchilega tubes were about
$0.6 \mathrm{~cm}$ in diameter, and the wall of the corers was $0.1 \mathrm{~cm}$ thick. Hence, the smallest corer sampled sediment at a distance of 0 to $0.6 \mathrm{~cm}$ from the $L$. conchilega tube, the second corer at a distance of 0.7 to $1.3 \mathrm{~cm}$ and the largest at a distance of 1.4 to $2.4 \mathrm{~cm}$. The sediment of each corer was washed through a $0.5 \mathrm{~mm}$ mesh sieve and the number of $U$. poseidonis determined. In addition, 17 single tubes of $L$. conchilega without surrounding sediment were collected and examined for amphipod inhabitants.

Laboratory experiment with Urothoe poseidonis. The field studies led to the hypotheses that, unlike other amphipods, $U$. poseidonis may not regularly migrate into the water column, but remain in the sediment for long periods of time.

To examine the migrating and swimming activity of Urothoe poseidonis a laboratory experiment was carried out. A tank was filled with a $4 \mathrm{~cm}$ layer of sand and connected to a slow but continuous seawater inflow. Outlets consisted of 4 tubes with a diameter of $0.5 \mathrm{~cm}$, which drained the water into a second tank. The outlet of the second tank was a $1 \times 10 \mathrm{~cm}$ hole in the wall of the tank covered with $0.5 \mathrm{~mm}$ gauze. Amphipods were released into the first

Table 1. Sampling schedule for Lanice conchilega tubes and associated fauna in Rhossili Bay, UK. Number of replicates is given (core samples covering $32 \mathrm{~cm}^{2}$ ) for 3 categories: samples with 2 to $5 \mathrm{~L}$. conchilega tubes, samples with 1 tube and reference samples with no tubes

\begin{tabular}{|lrcc|}
\hline Sampling date & $2-5$ tubes & 1 tube & 0 tubes \\
\hline 1998 & & & \\
26 May & 6 & 3 & 7 \\
24 Jun & 3 & 13 & 8 \\
24 Jul & 11 & 5 & 8 \\
8 Aug & 8 & 8 & 8 \\
10 Oct & 10 & 6 & 8 \\
4 Nov & 8 & 8 & 8 \\
4 Dec & 7 & 9 & 8 \\
1999 & & & \\
19 Jan & 10 & 6 & 8 \\
15 Feb & 8 & 8 & 8 \\
20 Mar & 8 & 8 & 8 \\
4 Apr & 8 & 8 & 8 \\
\hline
\end{tabular}


tank, and it was checked that individuals swam around actively and dug themselves into the sediment, and that they were not passively drained into the second tank. Amphipods that actively swam into the second tank were trapped and could not return to the first tank.

On Day 1 of the experiment, 15 individuals of Urothoe poseidonis were released into each of 3 parallel set-ups. As a control, 15 individuals of the haustoriid amphipod Bathyporeia pelagica were inserted into each of 3 experimental set-ups identical to those used for $U$. poseidonis. The tanks were checked regularly for trapped amphipods for $9 \mathrm{~d}$. A second experiment was set up identical to the first, but with Atylus swammerdamei as the control species (10 individuals per tank). For this experiment the number of trapped amphipods in Tank 2 was checked for $41 \mathrm{~d}$.

Sediment analyses. To investigate potential effects of Lanice conchilega tubes on the grain size composition of the sediment, surface samples were taken in close proximity to groups of $L$. conchilega (2 to 5 tubes), to single L. conchilega tubes, and from intermediate tube-free sand (corer: $26 \mathrm{~mm}$ diameter, $2 \mathrm{~cm}$ deep; sampling dates: 4 December 1998, 15 February 1999, 14 April 1999). On each date, 5 samples from each of the 3 categories were taken and pooled. The samples were analysed with a Coulter ${ }^{\circledR}$ LS particle size analyzer at the University of Gent (Belgium). Since this analyzer only analyses grain sizes up to $800 \mu \mathrm{m}$, coarser fractions were separated, sieved through a set of 2, 1 and $0.85 \mathrm{~mm}$ mesh sieves, and weighed.

Data analyses. The benthic community structure was analysed with the PRIMER statistical package (Clarke \& Warwick 1994). The term 'community' was used for species assemblages in the samples. A species assemblage composed of Lanice conchilega tubes plus accompanying organisms was designated a 'L. conchilega community', whereby interactions between species were not implied.

The data matrix of all benthic species was 4th-root transformed in order to down-weight abundant species. The Bray-Curtis index was calculated between each possible pair of samples. A 2-way analysis of similarity (ANOSIM) was used to simultaneously test for significant temporal differences and those between the Lanice conchilega categories (2 to 5 tubes, 1 tube, no tubes). A 1-way ANOSIM was used to test between the L. conchilega categories on a monthly base.

Results were visualised by non-parametric multidimensional scaling (MDS). Species mainly responsible for differences between Lanice conchilega and reference communities were identified with the PRIMER programme SIMPER. This examines the percentage contribution each species makes to the similarity within and dissimilarity between communities.
In order to simultaneously assess the effect of the Lanice conchilega categories and time on species richness and abundance, a 2-way ANOVA was attempted. However, variances were not homogeneous, even after data transformation. Both factors were then tested separately with the non-parametric Kruskal-Wallis test $(p<0.05)$. Tests were performed on the unbalanced numbers of replicates, which can potentially lead to difficulties with the analyses. Hence, numbers of replicates were balanced to 8 replicates per L. conchilega category and month by randomly deleting values from categories with more than 8 replicates and replacing missing data with mean values for the respective category (Underwood 1997). The resulting significance values of the Kruskal-Wallis tests were identical to those of the tests of the unbalanced data with 1 exception (see 'Results; Species richness and total abundance'). A Mann-Whitney $U$-test was applied as the pairwise post-hoc test to the original, unbalanced data $(\mathrm{p}<0.05)$.

\section{RESULTS}

\section{Sediment}

The sediment of Rhossili Bay consisted of 85 to $99 \%$ of fine and very fine sand. On 2 of the 3 dates when sediment samples were taken, small fractions of coarser material (850 to $2000 \mu \mathrm{m}$ ) were found in samples collected around Lanice conchilega tubes (15 February 1999: 0.6 to $0.9 \%$; 14 April 1999: 1.4 to $4.5 \%$ ). No other conspicuous differences in grain size distribution were found between samples from around L. conchilega tubes and reference samples.

\section{Species richness and total abundance}

Altogether, 53 species were found in samples with Lanice conchilega tubes and 29 in reference samples with no L. conchilega (Table 2); 27 species were exclusively found in samples with $L$. conchilega tubes. Altogether, 12 individuals of the scaleworms Harmothoe imbricata and $H$. lunulata were found inside L. conchilega tubes.

Several isopod and amphipod species were among those found exclusively in samples with Lanice conchilega tubes (Pontocrates arenarius, Eurydice pulchra, Idotea emarginata, I. linearis, I. metallica, I. pelagica, Bathyporeia sarsi), but most were single recordings. Juvenile Mytilus edulis, Harmothoe lunulata, juvenile Carcinus meanas, Gammarus locusta and Tanaissus lilljeborgi were recorded on 3 or more dates in samples containing $L$. conchilega tubes, but never in reference samples. 
Table 2. Presence (x) and absence (-) of infauna species in samples with and without Lanice conchilega tubes from May 1998 (M) to April 1999 (A)

\begin{tabular}{|c|c|c|c|c|c|c|c|c|c|c|c|c|c|c|c|c|c|c|c|c|c|c|}
\hline \multirow[t]{2}{*}{ Taxon } & \multicolumn{11}{|c|}{ Samples with tubes } & \multicolumn{11}{|c|}{ Samples with no tubes } \\
\hline & $\mathrm{M}$ & $\mathrm{J}$ & $\mathrm{J}$ & A & $\mathrm{O}$ & $\mathrm{N}$ & $\mathrm{D}$ & $\mathrm{J}$ & $\mathrm{F}$ & $\mathrm{M}$ & A & $\mathrm{M}$ & $\mathrm{J}$ & $\mathrm{J}$ & A & $\mathrm{O}$ & $\mathrm{N}$ & $\mathrm{D}$ & $\mathrm{J}$ & $\mathrm{F}$ & $\mathrm{M}$ & A \\
\hline \multicolumn{23}{|l|}{ Bivalvia } \\
\hline Angulus tenuis & $\mathrm{x}$ & $\mathrm{x}$ & - & - & - & $\mathrm{x}$ & $\mathrm{x}$ & - & - & - & - & - & $\mathrm{x}$ & - & - & - & - & $\mathrm{x}$ & - & - & - & - \\
\hline Mysella bidentata & - & - & $\mathrm{x}$ & _- & - & _- & - & - & - & $\mathrm{x}$ & - & - & - & - & - & _- & - & _- & - & - & - & - \\
\hline Mytilus edulis & $\mathrm{x}$ & - & - & $\mathrm{x}$ & $\mathrm{x}$ & $\mathrm{x}$ & - & - & - & - & $\mathrm{x}$ & - & - & - & - & - & - & - & - & - & - & - \\
\hline Tellimya ferruginosa & - & - & - & - & $\mathrm{x}$ & - & - & - & - & - & - & $\mathrm{x}$ & - & - & - & - & - & - & - & - & - & - \\
\hline \multicolumn{23}{|l|}{ Polychaeta } \\
\hline Capitalla capitata & - & $\mathrm{x}$ & $\mathrm{x}$ & $\mathrm{x}$ & $\mathrm{x}$ & $\mathrm{x}$ & $\mathrm{x}$ & $\mathrm{x}$ & $\mathrm{x}$ & $\mathrm{x}$ & - & - & - & $\mathrm{x}$ & - & $\mathrm{x}$ & $\mathrm{x}$ & $\mathrm{x}$ & $\mathrm{x}$ & $\mathrm{x}$ & - & - \\
\hline Chaetozone setosa & - & - & - & - & - & - & - & - & $\mathrm{x}$ & - & - & - & - & - & - & - & - & - & - & - & - & - \\
\hline Eteone spetsbergensis & - & - & - & - & - & - & - & - & - & - & - & - & - & - & - & $\mathrm{x}$ & - & - & - & - & - & - \\
\hline Eumida sanguinea & $\mathrm{x}$ & $\mathrm{x}$ & $\mathrm{x}$ & $\mathrm{x}$ & $\mathrm{x}$ & $\mathrm{x}$ & $\mathrm{x}$ & $\mathrm{x}$ & $\mathrm{x}$ & $\mathrm{x}$ & $\mathrm{x}$ & - & $\mathrm{x}$ & - & $\mathrm{x}$ & $\mathrm{x}$ & - & - & $\mathrm{x}$ & - & - & $\mathrm{x}$ \\
\hline Eunoe nodosa & $\mathrm{x}$ & - & - & - & - & - & - & - & - & - & - & - & - & - & - & - & - & - & - & - & - & - \\
\hline Glycera alba & - & - & - & - & - & - & - & - & - & - & - & - & - & - & - & - & - & - & - & $\mathrm{x}$ & - & - \\
\hline Glycera tridactyla & - & - & - & - & - & - & - & - & - & - & - & $\mathrm{x}$ & - & - & - & - & - & - & - & - & - & - \\
\hline Harmothoe imbricata & $\mathrm{x}$ & - & - & - & - & - & - & - & - & - & - & - & - & - & - & - & - & - & - & - & - & - \\
\hline Harmothoe lunulata & $\mathrm{x}$ & - & - & - & $\mathrm{x}$ & $\mathrm{x}$ & - & $\mathrm{x}$ & - & - & $\mathrm{x}$ & - & - & - & - & - & - & - & - & - & - & - \\
\hline Hediste diversicolor & - & - & - & - & - & - & $\mathrm{x}$ & - & - & - & - & - & - & - & - & - & - & - & - & - & - & - \\
\hline Lanice conchilega & $\mathrm{x}$ & $\mathrm{x}$ & $\mathrm{x}$ & $\mathrm{x}$ & $\mathrm{x}$ & $\mathrm{x}$ & $\mathrm{x}$ & $\mathrm{x}$ & $\mathrm{x}$ & $\mathrm{x}$ & $\mathrm{x}$ & - & - & - & - & - & - & - & - & - & - & - \\
\hline Magelona mirabilis & $\mathrm{x}$ & - & - & - & - & $\mathrm{x}$ & $\mathrm{x}$ & - & $\mathrm{x}$ & - & $\mathrm{x}$ & - & $\mathrm{x}$ & - & - & $\mathrm{x}$ & $\mathrm{x}$ & $\mathrm{x}$ & $\mathrm{x}$ & $\mathrm{x}$ & - & - \\
\hline Malacoceros fuliginosus & $\mathrm{x}$ & $\mathrm{x}$ & - & - & - & - & - & - & - & - & - & $\mathrm{x}$ & - & - & - & - & - & - & - & - & - & - \\
\hline Malacoceros tetracerus & - & - & - & - & $\mathrm{x}$ & - & - & $\mathrm{x}$ & - & - & - & - & - & - & - & $\mathrm{x}$ & - & - & - & - & - & - \\
\hline Malacoceros vulgaris & - & - & - & - & $\mathrm{x}$ & - & - & - & - & - & - & - & - & - & - & - & - & - & - & - & - & - \\
\hline Neanthes fucata & - & - & - & - & - & - & - & $\mathrm{x}$ & - & - & - & - & - & - & - & - & - & - & - & - & - & - \\
\hline Nephtys caeca & - & $\mathrm{x}$ & - & $\mathrm{x}$ & $\mathrm{x}$ & $\mathrm{x}$ & $\mathrm{x}$ & $\mathrm{x}$ & $\mathrm{x}$ & $\mathrm{x}$ & $\mathrm{x}$ & - & $\mathrm{x}$ & - & $\mathrm{x}$ & $\mathrm{x}$ & $\mathrm{x}$ & $\mathrm{x}$ & $\mathrm{x}$ & $\mathrm{x}$ & $\mathrm{x}$ & $\mathrm{x}$ \\
\hline Nephtys cirrosa & $\mathrm{x}$ & $\mathrm{x}$ & - & - & - & - & - & - & $\mathrm{x}$ & - & $\mathrm{x}$ & $\mathrm{x}$ & $\mathrm{x}$ & - & - & - & - & - & - & - & - & - \\
\hline Nephtys hombergi & - & - & - & - & - & - & - & $\mathrm{x}$ & - & $\mathrm{x}$ & - & - & - & - & - & - & - & - & - & - & - & - \\
\hline Orbinia sertulata & - & $\mathrm{x}$ & - & - & - & - & $\mathrm{x}$ & - & $\mathrm{x}$ & - & - & - & - & - & - & - & - & - & $\mathrm{x}$ & $\mathrm{x}$ & - & $\mathrm{x}$ \\
\hline Owenia fusiformis & $\mathrm{x}$ & $\mathrm{x}$ & $\mathrm{x}$ & $\mathrm{x}$ & $\mathrm{x}$ & $\mathrm{x}$ & $\mathrm{x}$ & $\mathrm{x}$ & $\mathrm{x}$ & $\mathrm{x}$ & $\mathrm{x}$ & $\mathrm{x}$ & $\mathrm{x}$ & $\mathrm{x}$ & $\mathrm{x}$ & $\mathrm{x}$ & $\mathrm{x}$ & $\mathrm{x}$ & $\mathrm{x}$ & $\mathrm{x}$ & $\mathrm{x}$ & $\mathrm{x}$ \\
\hline Phyllodoce mucosa & $\mathrm{x}$ & $\mathrm{x}$ & - & - & $\mathrm{x}$ & $\mathrm{x}$ & - & $\mathrm{x}$ & $\mathrm{x}$ & $\mathrm{x}$ & $\mathrm{x}$ & - & - & - & - & - & - & - & $\mathrm{x}$ & - & - & $\mathrm{x}$ \\
\hline Sigalion mathildae & - & - & $\mathrm{x}$ & - & - & $\mathrm{x}$ & - & $\mathrm{x}$ & - & - & - & - & - & $\mathrm{x}$ & - & - & - & - & - & - & - & - \\
\hline Spio filicornis & - & $\mathrm{x}$ & - & - & - & - & - & $\mathrm{x}$ & - & - & - & - & - & - & - & - & - & - & - & - & - & $\mathrm{x}$ \\
\hline Spio martinensis & $\mathrm{x}$ & - & - & - & $\mathrm{x}$ & - & - & $\mathrm{x}$ & - & $\mathrm{x}$ & - & - & - & - & - & - & $\mathrm{x}$ & $\mathrm{x}$ & - & $\mathrm{x}$ & - & - \\
\hline \multicolumn{23}{|l|}{ Crustacea } \\
\hline Atylus swammerdamei & - & - & - & - & - & $\mathrm{x}$ & $\mathrm{x}$ & $\mathrm{x}$ & $\mathrm{x}$ & - & - & - & - & - & - & - & $\mathrm{x}$ & - & $\mathrm{x}$ & $\mathrm{x}$ & - & - \\
\hline Bathyporeia elegans & $\mathrm{x}$ & $\mathrm{x}$ & - & $\mathrm{x}$ & $\mathrm{x}$ & $\mathrm{x}$ & - & $\mathrm{x}$ & $\mathrm{x}$ & $\mathrm{x}$ & $\mathrm{x}$ & - & - & - & - & - & - & - & $\mathrm{x}$ & - & $\mathrm{x}$ & $\mathrm{x}$ \\
\hline Bathyporeia nana & - & - & - & $\mathrm{x}$ & - & - & - & - & - & - & - & - & - & - & - & - & - & - & - & - & - & - \\
\hline Bathyporeia pelagica & - & - & - & - & $\mathrm{x}$ & $\mathrm{x}$ & $\mathrm{x}$ & $\mathrm{x}$ & - & - & - & - & - & - & - & $\mathrm{x}$ & $\mathrm{x}$ & $\mathrm{x}$ & - & - & - & - \\
\hline Bathyporeia pilosa & - & - & - & - & - & - & - & $\mathrm{x}$ & $\mathrm{x}$ & $\mathrm{x}$ & - & - & - & - & - & - & - & - & $\mathrm{x}$ & $\mathrm{x}$ & - & - \\
\hline Bathyporeia sarsi & - & - & - & $\mathrm{x}$ & $\mathrm{x}$ & - & - & - & - & - & - & - & - & - & - & - & - & - & - & - & - & - \\
\hline Caprella linearis & - & - & - & - & - & - & - & - & - & $\mathrm{x}$ & - & - & - & - & - & - & - & - & - & - & - & - \\
\hline Carcinus maenas & $\mathrm{x}$ & $\mathrm{x}$ & $\mathrm{x}$ & - & - & - & - & - & - & - & $\mathrm{x}$ & - & - & - & - & - & - & - & - & - & - & - \\
\hline Corophium volutator & - & $\mathrm{x}$ & - & - & - & - & - & - & - & - & - & - & - & - & - & - & - & - & - & - & - & - \\
\hline Crangon crangon & - & $\mathrm{x}$ & - & $\mathrm{x}$ & - & - & - & - & - & - & - & - & - & - & $\mathrm{x}$ & - & - & - & - & - & - & - \\
\hline Eurydice pulchra & - & - & - & $\mathrm{x}$ & - & - & - & - & - & - & - & - & - & - & - & - & - & - & - & - & - & - \\
\hline Gammarus locusta & - & - & - & - & $\mathrm{x}$ & $\mathrm{x}$ & - & - & - & - & $\mathrm{x}$ & - & - & - & - & - & - & - & - & - & - & - \\
\hline Gastrosaccus spinifer & - & - & - & - & - & $\mathrm{x}$ & - & - & - & - & - & - & - & - & - & - & $\mathrm{x}$ & - & - & - & - & - \\
\hline Idotea emarginata & - & - & - & $\mathrm{x}$ & - & - & - & - & - & - & - & - & - & - & - & - & - & - & - & - & - & - \\
\hline Idotea linearis & - & $\mathrm{x}$ & - & - & - & - & - & - & - & - & - & - & - & - & - & - & - & - & - & - & - & - \\
\hline Idotea metallica & - & - & - & - & $\mathrm{x}$ & - & - & - & - & - & - & - & - & - & - & - & - & - & - & - & - & - \\
\hline Idotea pelagica & - & - & - & $\mathrm{x}$ & - & - & - & - & - & - & - & - & - & - & - & - & - & - & - & - & - & - \\
\hline Iphinoe trispinosa & $\mathrm{x}$ & - & - & $\mathrm{x}$ & - & - & - & - & - & - & - & - & - & - & - & - & - & - & - & - & - & - \\
\hline Microprotopus maculatus & - & - & - & $\mathrm{x}$ & $\mathrm{x}$ & - & - & - & - & - & - & - & - & - & - & - & - & $\mathrm{x}$ & - & - & - & - \\
\hline Pontocrates altamarinus & - & - & - & - & $\mathrm{x}$ & - & $\mathrm{x}$ & - & $\mathrm{x}$ & - & - & - & - & - & - & $\mathrm{x}$ & - & - & $\mathrm{x}$ & - & - & - \\
\hline Pontocrates arenarius & - & - & - & $\mathrm{x}$ & - & - & - & - & - & - & - & - & - & - & - & - & - & - & - & - & - & - \\
\hline Portumnus latipes & - & - & - & $\mathrm{x}$ & - & $\mathrm{x}$ & - & $\mathrm{x}$ & $\mathrm{x}$ & - & - & - & - & - & - & $\mathrm{x}$ & - & - & $\mathrm{x}$ & $\mathrm{x}$ & - & - \\
\hline Tanaissus lilljeborgi & - & - & - & $\mathrm{x}$ & - & $\mathrm{x}$ & $\mathrm{x}$ & - & - & $\mathrm{x}$ & - & - & - & - & - & - & - & - & - & - & - & - \\
\hline Urothoe marina & - & - & - & $\mathrm{x}$ & - & - & - & - & - & - & - & - & - & - & - & - & - & - & - & - & - & - \\
\hline Urothoe poseidonis & $\mathrm{x}$ & $\mathrm{x}$ & $\mathrm{x}$ & $\mathrm{x}$ & $\mathrm{x}$ & $\mathrm{x}$ & $\mathrm{x}$ & $\mathrm{x}$ & $\mathrm{x}$ & $\mathrm{x}$ & $\mathrm{x}$ & $\mathrm{x}$ & $\mathrm{x}$ & $\mathrm{x}$ & $\mathrm{x}$ & $\mathrm{x}$ & $\mathrm{x}$ & $\mathrm{x}$ & $\mathrm{x}$ & $\mathrm{x}$ & - & $\mathrm{x}$ \\
\hline \multicolumn{23}{|l|}{ Ophiuroidea } \\
\hline Amphiura brachiata & - & $\mathrm{x}$ & - & - & - & - & - & $\mathrm{x}$ & $\mathrm{x}$ & - & $\mathrm{x}$ & - & - & - & - & - & - & - & $\mathrm{x}$ & - & - & - \\
\hline Ophiura albida & - & $\mathrm{x}$ & - & - & - & - & - & - & - & - & - & - & - & - & - & - & - & - & - & - & - & - \\
\hline
\end{tabular}


Table 3. Lanice conchilega. Effect of tubes on infauna species richness and abundance (Kruskal-Wallis test). Factors are $L$. conchilega (2 to 5 tubes, 1 tube, no tubes) and time (i.e. month of sampling; 11 samples from May 1998 to April 1999)

\begin{tabular}{|llrcc|}
\hline Variable & Factor & $\mathrm{df}$ & $\chi^{2}$ & $\mathrm{p}$ \\
\hline Species richness & L. conchilega & 2 & 97.62 & $<0.0001$ \\
& Time & 10 & 37.2 & $<0.0001$ \\
& & & & \\
Abundance & L. conchilega & 2 & 107.86 & $<0.0001$ \\
& Time & 10 & 18.36 & 0.049 \\
\hline
\end{tabular}

Table 4. Lanice conchilega. Pairwise comparisons of effect of L. conchilega tube categories on infauna species richness and abundance (Mann-Whitney test)

\begin{tabular}{|lccc|}
\hline Variable & $\begin{array}{c}2-5 \text { tubes vs. } \\
0 \text { tubes }\end{array}$ & $\begin{array}{c}1 \text { tube vs. } \\
0 \text { tubes }\end{array}$ & $\begin{array}{c}2-5 \text { tubes vs. } \\
1 \text { tube }\end{array}$ \\
\hline Species richness & $<0.0001$ & $<0.0001$ & 0.867 \\
Abundance & $<0.0001$ & $<0.0001$ & 0.001 \\
\hline
\end{tabular}

The species richness and abundance of individuals differed significantly between the samples with and without Lanice conchilega tubes (Tables 3 \& 4). From May 1998 to April 1999, 4.9 \pm 1.8 species were found in samples with 2 to $5 \mathrm{~L}$. conchilega tubes (mean $\pm \mathrm{SD}$ $\left.32 \mathrm{~cm}^{-2}, \mathrm{n}=88\right), 4.8 \pm 1.8$ species in samples with 1 tube $(\mathrm{n}=81)$ and $2.4 \pm 1.3$ species in samples with no tubes $(\mathrm{n}=87)$. Abundances were highest in samples with 2 to $5 \mathrm{~L}$. conchilega tubes with $19.1 \pm 8.5$ individuals $32 \mathrm{~cm}^{-2}(\mathrm{n}=88)$. Samples with 1 tube had $15.4 \pm 7.7$ individuals $32 \mathrm{~cm}^{-2}$, and those with no tubes $7.4 \pm 4.3$ individuals $32 \mathrm{~cm}^{-2}$ (1 tube samples: $\mathrm{n}=88$; no tube samples: $n=87$ ). Pairwise tests showed that species richness was not significantly different between samples with 1 tube and those with 2 to 5 tubes, while abundance of individuals was significantly different between the 2 categories (Table 4 ).

During the year, species richness ranged between $3.1 \pm 1.5$ in March 1999 and $5.0 \pm 2.4$ in June 1998 (mean $\pm \mathrm{SD} 32 \mathrm{~cm}^{-2}, \mathrm{n}=24$ ). Time, i.e. month of sampling, had a significant effect on species richness (Table 3), but there was no clear seasonal trend. Pairwise tests of consecutive months showed significantly higher species richness in November than in December 1998 (Mann-Whitney test, $\mathrm{p}=0.047, \mathrm{n}=24$ for each month; November: $4.5 \pm 2.1$ species $32 \mathrm{~cm}^{-2}$; December: $3.5 \pm 1.4$ species $32 \mathrm{~cm}^{-2}$ ) and significantly higher values for February than for March 1999 (Mann-Whitney test, $\mathrm{p}=0.004, \mathrm{n}=24$ for each month; February: $5.0 \pm$ 2.2 species $32 \mathrm{~cm}^{-2}$; March: $3.1 \pm 1.5$ species $32 \mathrm{~cm}^{-2}$ ). The regular occurrence of the amphipods Atylus swammerdamei and Bathyporeia spp. were responsible for the higher numbers in February.
Time also had a significant influence on abundance of individuals (Table 3). However, running the same analyses (Kruskal-Wallis test) on a balanced set of data with dummy values for missing replicates produced a significance level of $p=0.063$ for abundance. Hence, the effect of time on abundance is uncertain.

\section{Community structure}

Integrated over the entire study period, the species community around Lanice conchilega tubes was significantly different from that in tube-free sediment (ANOSIM $\mathrm{p}<0.001$ for pairwise differences between samples with 1 or 2 to 5 tubes compared to tube-free samples). Communities in samples with 1 and 2 to 5 L. conchilega were also significantly different (ANOSIM, $p=0.05$ ). At the same time there was significant temporal change. The communities differed significantly between all possible combinations of sampling months (ANOSIM, $\mathrm{p}<0.006$ for pairwise comparisons) with 3 exceptions (May vs. June 1998, November vs. December 1998 and January vs. March 1999). The MDS ordination in Fig. 3 illustrates the temporal change. Although there is no clear grouping of individual months, winter and autumn months cluster on different sides of the MDS ordination than spring and summer months.

Tests of differences between the 3 categories with and without Lanice conchilega on a monthly basis revealed consistently significant differences between communities from samples with single or groups of $L$. conchilega and tube-free samples, with 3 exceptions (Table 5). Only once did samples with single tubes differ significantly from those with 2 to 5 tubes.

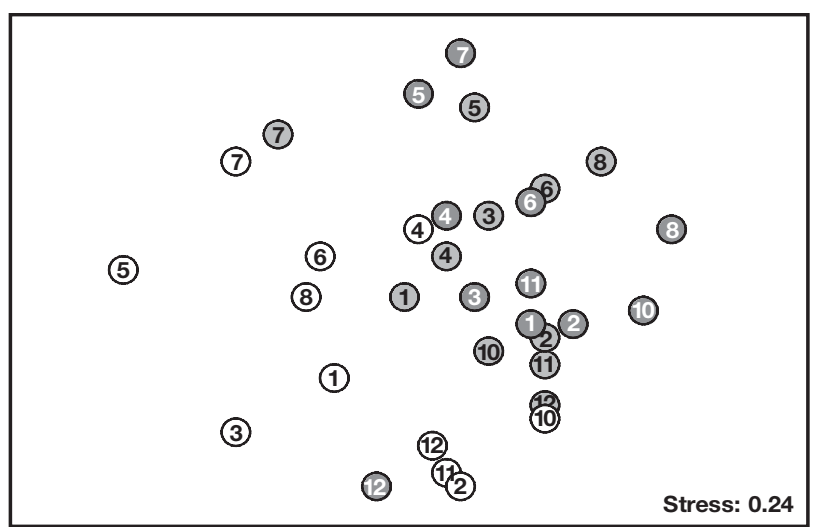

(1) Samples with 2-5 tubes

(n) Samples with 1 tube

(n) Samples with no tubes

Fig. 3. MDS ordination of similarities between infauna communities at Rhossili Bay from May 1998 to April 1999 in samples containing 2 to 5 Lanice conchilega tubes, samples containing 1 tube, and samples with no tubes (Bray-Curtis index, $\sqrt{ } \sqrt{ }$-transformation of mean abundance); numbers: month, e.g. 1: January 
Table 5. Significance levels for differences between infauna communities from samples with groups of Lanice conchilega tubes ( 2 to 5 tubes), 1 tube and samples with no tubes (ANOSIM). Significant differences $(p<0.05)$ in bold

\begin{tabular}{|cccc|}
\hline Date & $\begin{array}{c}2-5 \text { tubes vs. } \\
\text { no tubes }\end{array}$ & $\begin{array}{c}1 \text { tube vs. } \\
\text { no tubes }\end{array}$ & $\begin{array}{c}2-5 \text { tubes vs. } \\
1 \text { tube }\end{array}$ \\
\hline 1998 & & & \\
May & $\mathbf{0 . 0 0 6}$ & $\mathbf{0 . 0 3 3}$ & 0.643 \\
Jun & $\mathbf{0 . 0 0 6}$ & $\mathbf{0 . 0 0 1}$ & 0.866 \\
Jul & $\mathbf{0 . 0 0 1}$ & 0.238 & $\mathbf{0 . 0 0 2}$ \\
Aug & $\mathbf{0 . 0 0 1}$ & $\mathbf{0 . 0 0 1}$ & 0.102 \\
Oct & $\mathbf{0 . 0 0 1}$ & 0.158 & 0.282 \\
Nov & $\mathbf{0 . 0 0 1}$ & $\mathbf{0 . 0 0 3}$ & 0.056 \\
Dec & $\mathbf{0 . 0 0 3}$ & $\mathbf{0 . 0 0 1}$ & 0.464 \\
1999 & & & \\
Jan & $\mathbf{0 . 0 0 1}$ & $\mathbf{0 . 0 0 9}$ & 0.122 \\
Feb & $\mathbf{0 . 0 0 1}$ & $\mathbf{0 . 0 0 1}$ & 0.088 \\
Mar & $\mathbf{0 . 0 0 1}$ & $\mathbf{0 . 0 0 1}$ & 0.501 \\
Apr & 0.765 & $\mathbf{0 . 0 3 3}$ & 0.261 \\
\hline
\end{tabular}

Throughout the study, the polychaete Eumida sanguinea and the amphipod Urothoe poseidonis were primarily responsible for the differences between samples with groups or single Lanice conchilega and reference samples (Fig. 4). Furthermore, the amphipod species Atylus swammerdamei and amphipods of the genus Bathyporeia contributed considerably to the differences between communities. A. swammerdamei was responsible for up to $22 \%$ of the difference between groups of $L$. conchilega and reference samples without L. Conchilega (November 1998, December 1998, February 1999). On 5 of 11 sampling days, Bathyporeia spp. were responsible for over $10 \%$ of the difference between samples with no $L$. conchilega and samples with groups of L. conchilega (B. pelagica: October 1998, December 1998; B. sarsi: August 1998; B. elegans: March 1999). Other main contributors to differences between the communities were the amphipod species Micropotopus maculatus and the predatory polychaete species Phyllodoce mucosa, Nephtys caeca and $N$. cirrosa. The mean abundance of all these species was always higher in samples with L. conchilega than in samples without the tubeworm.

Apart from Lanice conchilega, another tube dwelling polychaete was regularly recorded (Owenia fusiformis). Its tubes are entirely buried in the sediment and do not leave any visible trace on the sediment surface. It was tested whether the benthic community structure was related to the numbers of $O$. fusiformis, but no significant relationship was found (PRIMER program RELATE, Spearman rank correlation, $p=0.175)$. Species richness was also found not to be significantly related to the abundance of $O$. fusiformis (Pearson correlation coefficient, $\mathrm{n}=184, \mathrm{p}=0.553)$.

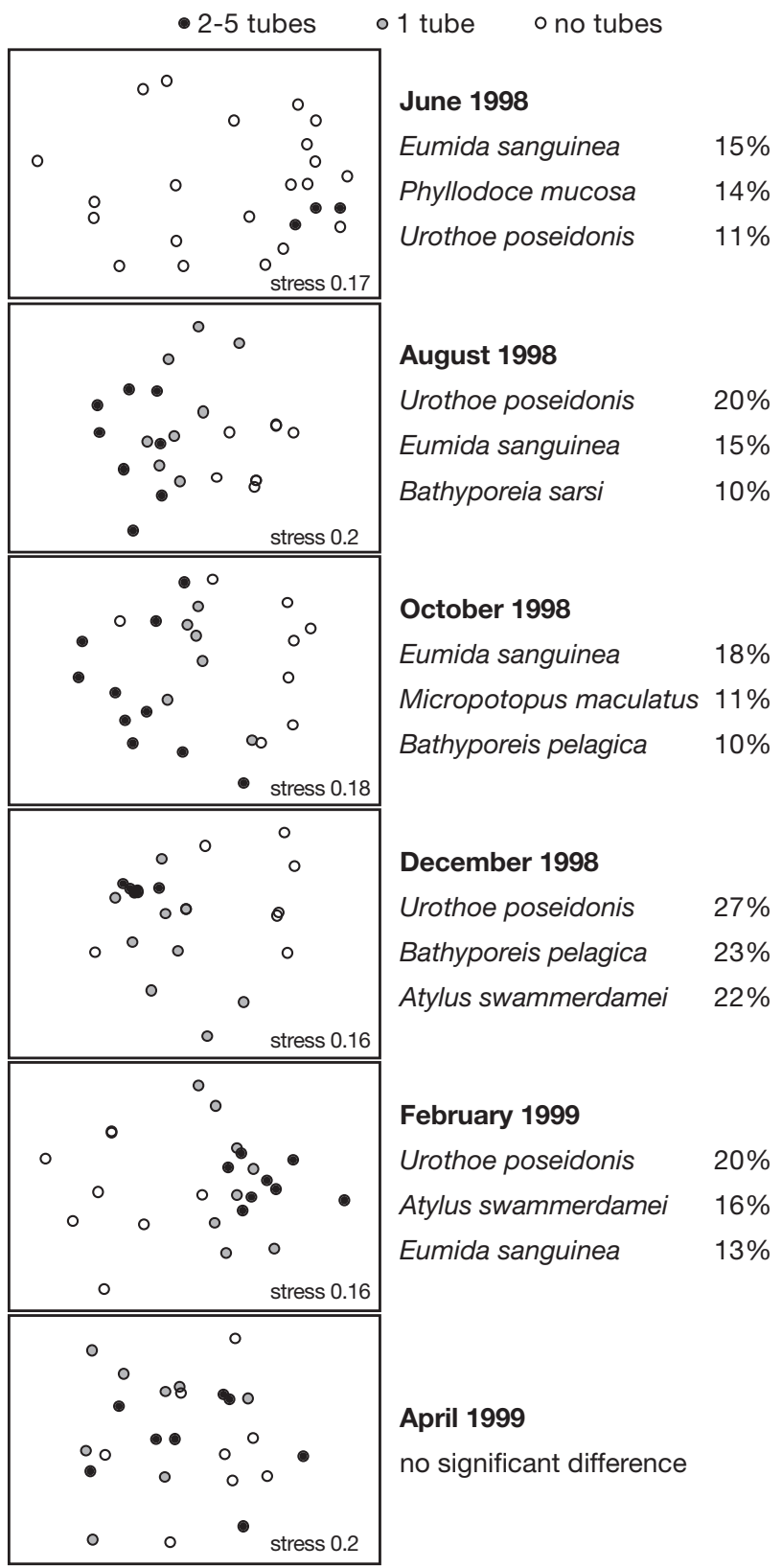

Fig. 4. MDS ordination of infauna communities in 2 mo intervals, grouped according to number of Lanice conchilega tubes. Significance levels for pairwise comparisons between communities are shown in Table 5. Species contributing most to dissimilarity between samples containing 2 to 5 tubes and samples with no tubes are shown, as well as their percentage contribution to dissimilarity (SIMPER)

\section{Abundance of individual species}

Since Eumida sanguinea, Atylus swammerdamei and Urothoe poseidonis were primarily responsible for the differences in community structure, their abundances were tested separately (Fig. 5). E. sanguinea was significantly more abundant in samples with Lanice conchi- 

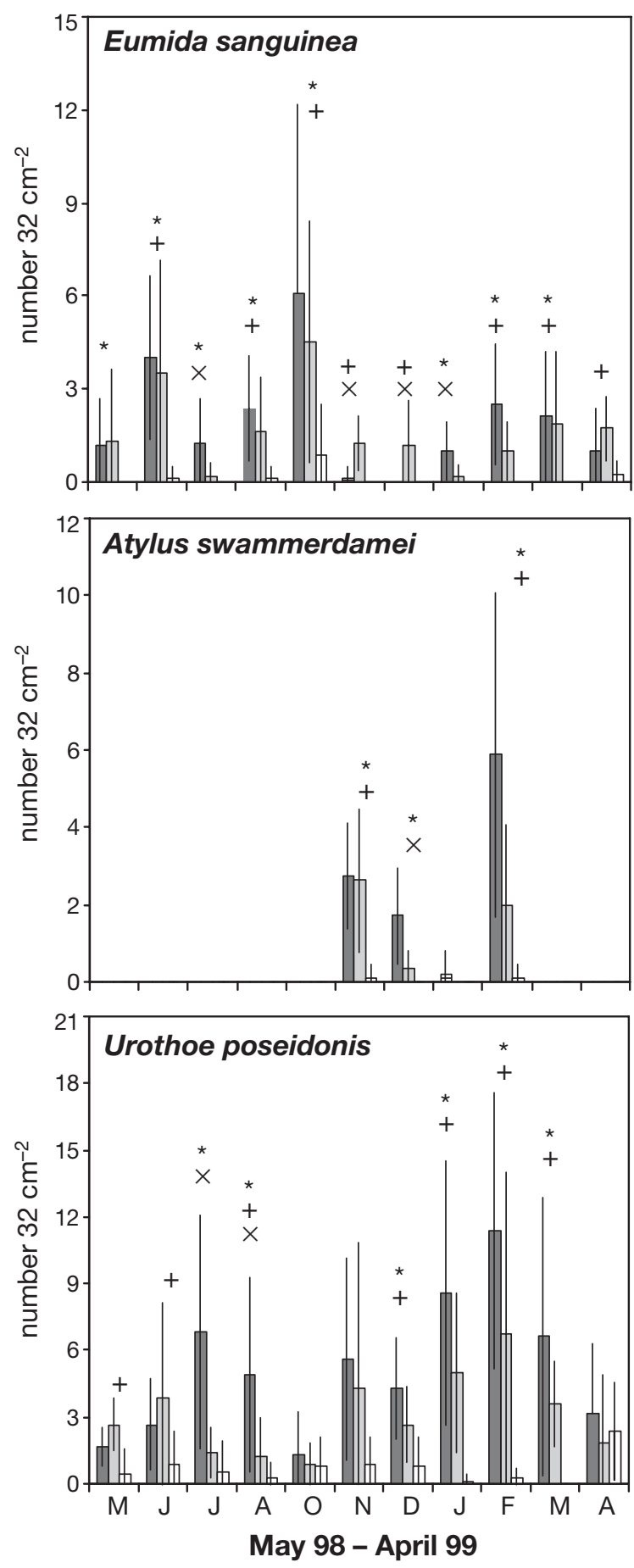

2-5 tubes $\square$ 1 tube $\square$ no tubes

$$
\begin{aligned}
& \text { * } 2-5 \text { tubes / 'notubes' }(p<0.05) \\
& +1 \text { tube / 'notubes' }(p<0.05) \\
& \times \quad 2-5 \text { tubes / } 1 \text { tube }(p<0.05)
\end{aligned}
$$

Fig. 5. Mean $( \pm \mathrm{SD})$ abundance of 3 infauna species in samples with 2 to 5 Lanice conchilega tubes, 1 tube and samples with no tubes. Significant differences are indicated lega throughout the study. This polychaete was almost exclusively found among the sand filaments of the topend fringe of $L$. conchilega tubes. In 8 out of 11 months U. poseidonis was significantly more abundant in samples with L. conchilega. A. swammerdamei was only present in Rhossili Bay in winter from November until February, and in 3 out of the 4 months its abundance was significantly higher in samples with $L$. conchilega tubes.

Other species were significantly more abundant in Lanice conchilega samples just once or twice during the study period: Phyllodoce mucosa (June), Nephtys cirrosa (June), Micropotopus maculatus (August and October), Bathyporeia elegans (November), Bathyporeia pelagica (December), Bathyporeia pilosa (February).

\section{Urothoe poseidonis and Lanice conchilega}

Most Urothoe poseidonis were females. Of a total of 693 individuals recorded from May 1998 to April 1999, only $6.5 \%$ were males. These amphipods were between 0.5 and $5 \mathrm{~mm}$ long. Throughout the year, 49 to $71 \%$ of individuals fell into the 1 to $2 \mathrm{~mm}$ size class, except for May and June 1998, when larger females of 3 to $5 \mathrm{~mm}$ in length were found with eggs or juveniles in their brood pouch and only $31 \%$ of the population were smaller individuals of 1 to $2 \mathrm{~mm}$.

While the majority of benthic animals inhabited the upper 0 to $4 \mathrm{~cm}$ of the sediment, Urothoe poseidonis was found deeper in the sediment. This amphipod lived at a depth of $4 \mathrm{~cm}$ or below, down to about $15 \mathrm{~cm}$ (Fig. 6). In contrast, other amphipod species, such as Bathyporeia pelagica, were found exclusively in the upper 0 to $2 \mathrm{~cm}$ surface layers of the sand.

No individual Urothoe poseidonis was found inside a Lanice conchilega tube; all inhabited the sediment in close proximity to the tube. On average, $2.0 \pm 3.0 \mathrm{U}$. poseidonis $23 \mathrm{~cm}^{-2}$ were found in the aggregated nested corers around individual $L$. conchilega tubes (equivalent to $2.9 \pm 4.1$ individuals $32 \mathrm{~cm}^{-2}$, the corer size used in the study of effects of $L$. conchilega on the community: mean $\pm \mathrm{SD}, \mathrm{n}=22$ ). In total, $4 U$. poseidonis were found in the smallest corers sampling the distance 0 to $0.6 \mathrm{~cm}$ around L. conchilega tubes (sum of 22 samples; $2.6 \pm 5.6$ individuals $\left.32 \mathrm{~cm}^{-2}\right)_{;} 28$ were found at a distance of 0.7 to $1.3 \mathrm{~cm}$ (sum of 22 samples; $7.4 \pm 13.1$ individuals $32 \mathrm{~cm}^{-2}$ ); and 13 were found at a distance of 1.4 to $2.4 \mathrm{~cm}$ (sum of 22 samples; $1.3 \pm 3.3$ individuals $32 \mathrm{~cm}^{-2}$ ).

\section{Swimming experiment with Urothoe poseidonis}

The laboratory experiments showed that in comparison with other amphipod species Urothoe poseidonis is not an active swimmer. Both control species Atylus 


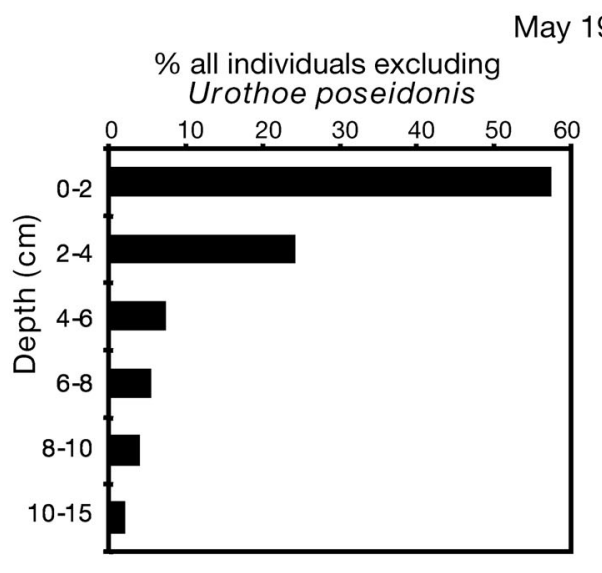

\section{$\%$ Urothoe poseidonis}

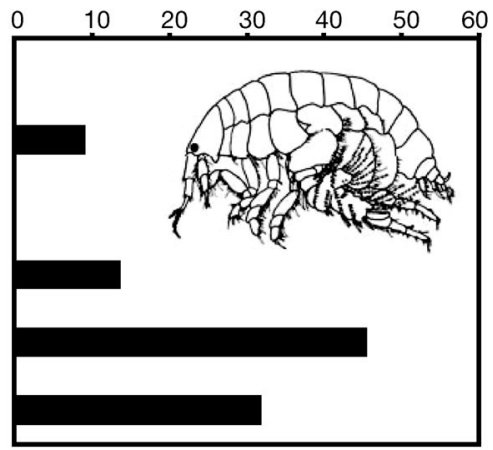

July 1998

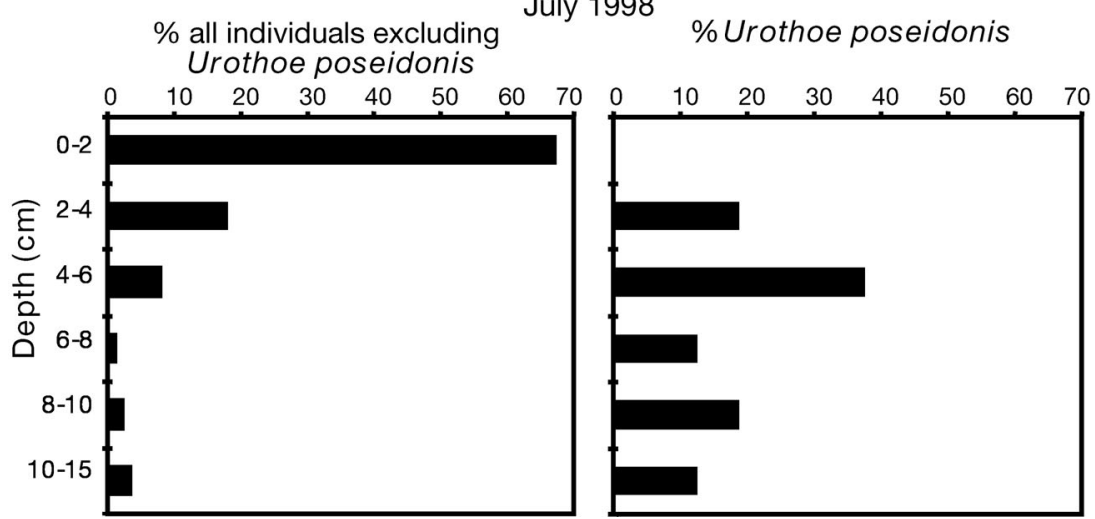

Fig. 6. Vertical distribution of benthic fauna in samples with single Lanice conchilega tubes. Number of all individuals excluding Urothoe poseidonis was 54 in May and 89 in July; number of $U$. poseidonis was 22 in May and 16 in July
Talman et al. 2004). Tubeworms are among such habitat creating species, and at high densities are known to change the benthic community structure. Sabellaria spp. for example create reefs of rigid tubes cemented together, which host a diverse fauna (Dubois et al. 2002). However, even small polychaetes that barely extend above the sediment surface, such as Pygospio elegans, affect the composition of the other infauna when they occur in dense aggregations (Bolam \& Fernandes 2003).

In the present study the tube worm Lanice conchilega was investigated. While past investigations have shown that tube lawns of $L$. conchilega with densities between 1500 and 4000 individuals $\mathrm{m}^{-2}$ change the benthic community by facilitating the settlement of bivalves and hosting higher numbers of predatory polychaetes (Zühlke 2001, Callaway 2003b), the present study has demonstrated that even single tubes are capable of generating an environment more beneficial to other benthic species. Small groups of 2 to 5 tubes had a greater effect on the community, but single tubes also had measurable effects on individual species as well as the entire community.

swammerdamei and Bathyporeia pelagica swam actively around the tanks into which they were released, only digging themselves into the sediment for short periods of time. Most individuals swam into the second tank on the first day of the experiment (Fig. 7). Of the 10 A. swammerdamei in each tank, 6 to 8 individuals migrated to the second tank on the same day the experiment started, and of the $15 \mathrm{~B}$. pelagica 10 to 13 individuals were found in the second tank after $2 \mathrm{~d}$.

Only a single Urothoe poseidonis swam into the second tank in each experiment. During the experiments it was checked that the $U$. poseidonis remained alive and active. The sediment in Tank 1 was stirred and the U. poseidonis flushed out. When the sediment was allowed to settle, all individuals responded by frantically shoveling sediment with the 5th enlarged pereiopod and rapidly digging themselves into the sediment again.

\section{DISCUSSION AND CONCLUSIONS}

Species that create prominent, complex 3-dimensional structures on the seabed change the composition of the benthic fauna (e.g. Diaz et al. 2003, Bomkamp et al. 2004,
The benthic fauna was not only influenced by the presence or absence of Lanice conchilega, but the community structure and species richness in Rhossili Bay changed significantly over time, and it cannot be ruled out that other, unmeasured factors such as the variable impact of waves and currents shaped the fauna. However, the study has shown that there is no seasonal limit to the effects of L. conchilega. Throughout the year, this tube dweller has significant effects on the community structure as well as on individual species.

Although most species utilise a specific feature of a habitat structuring organism, there are general functions of 3-dimensional structures that mainly affect 4 environmental components: (1) they improve the availability of attachment surfaces; (2) sediment properties are altered (e.g. grain size composition or porosity); (3) the hydrodynamic regime is modified (e.g. buffering of currents or increase of turbulence); (4) refuge from predators is created. The relevance of these factors depends on the individual characteristics of the habitat structuring species. For single Lanice conchilega tubes and small groups of 2 to 5 tubes, some of these effects are relatively weak. Single tubes provide little attachment surface for sessile species, 


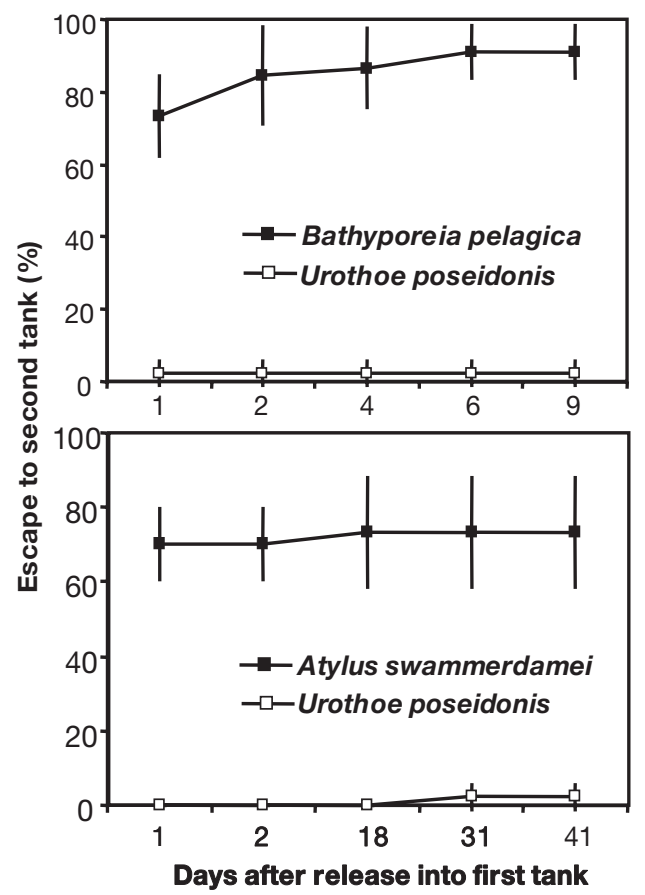

Fig. 7. Urothoe poseidonis. Tank experiment testing swimming activity. Amphipods Bathyporeia pelagica and Atylus swammerdamei served as control species. Percentages of amphipods that swam from the release tank to the second tank are shown $(\mathrm{n}=15 \mathrm{U}$. poseidonis, $15 \mathrm{~B}$. pelagica, 10 A. swammer damei)

especially on exposed shores. An animal settling on a L. conchilega tube top is in danger of being detached, or the entire L. conchilega tube top may be torn off by strong currents and waves.

In terms of sediment composition, small fractions of coarser sediment were found twice in close vicinity to Lanice conchilega tubes, but generally the presence of single $L$. conchilega tubes did not notably change the grain size distribution. However, immediately around the tubes, the sediment was regularly eroded and tubes were surrounded by small troughs, probably generated by water movement. The critical tube density that determines whether they have stabilising or destabilising effects on the sediment varies between species and depends on current velocity, but in general single tubes destabilise the sediment while dense tube lawns lead to sedimentation (Eckman et al. 1981, Luckenbach 1986, Carey 1987, Friedrichs et al. 2000).

The loosened sediment around single tubes may facilitate amphipod and isopod penetration of the sediment surface, explaining their higher numbers in samples with Lanice conchilega tubes. Many amphipods and isopods constantly migrate between the water column and the sediment (Fincham 1970), and a more fluid sediment may aid the process. Lackschewitz \& Reise (1998) reported maximum num- bers of Bathyporeia sarsi in faecal mounds of Arenicola marina, possibly for a similar reason.

Single Lanice conchilega tubes offer little refuge from predation: only the inside of the tube offers some shelter. Scaleworms Harmothoe spp. were found inside the tubes, and probably used them for protection. Harmothoe species are known to live commensally with other species that possess burrows and cavities (Hartmann-Schröder 1996).

The 2 species which benefited most from the presence of Lanice conchilega (the polychaete Eumida sanguinea and the amphipod Urothoe poseidonis) do not appear to have been affected by any of the above factors. E. sanguinea was mainly found among the sand fringe at the top of the tube. L. conchilega uses the fringe to trap suspended food particles and E. sanguinea may share this food source, which would make it a commensal in the true sense of the word. Most E. sanguinea in the tube fringes were small (between 0.3 and $2 \mathrm{~cm}$ long) and were probably juveniles. Adults attain a size of up to $9 \mathrm{~cm}$ (Hartmann-Schröder 1996). Given that only a few E. sanguinea were found in the tube-free sediment, L. conchilega tube fringes may constitute miniature hatcheries for young E. sanguinea, providing food and possibly some shelter.

Closer investigations into the relationship between the haustoriid amphipod Urothoe poseidonis and Lanice conchilega revealed that unlike the other amphipod species which live close to the sediment surface, $U$. poseidonis inhabits relatively deep sediment layers in close vicinity to the $L$. conchilega tubes. U. poseidonis is well adapted to digging sand: the carpus of Pereiopod 5 is at least twice as broad as it is long, and these shovel-like appendages are used to great effect when the amphipods dig themselves in (pers. obs.). Furthermore, the laboratory experiment indicated that in contrast to the other amphipod species $U$. poseidonis does not regularly migrate between the water column and the sediment, but remains buried. U. poseidonis is probably adapted to a permanent benthic life and does not usually leave the sediment, although males have occasionally been recorded in the water column (Salvat 1967, Vader 1978). Reasons for the diurnal migration of amphipods into the water column are likely to be associated with their reproductive cycle (Fincham 1970) and it can only be speculated that $U$. poseidonis has developed a different reproductive strategy, which allows it to remain buried in the sediment.

The actual benefit for Urothoe poseidonis living close to Lanice conchilega tubes may be an improved oxygen supply. According to Forster \& Graf (1995), when moving inside its tube $L$. conchilega acts as a piston, exchanging burrow water with the overlying water. Forster \& Graf (1995) found oxygen at all depths in adjacent sediment along the tube. U. poseidonis has 
also been reported to live in association with Arenicola marina, Echinocardium cordatum, Acrochnida brachiata and Balanoglossus sp. (Crawford 1937: cited in Salvat 1967, Vader 1978, Lackschewitz \& Reise 1998). Lackschewitz \& Reise (1998) suggested that $U$. poseidonis benefited from the steady food and oxygen supply and the minor temperature fluctuations inside lugworm burrows. They reasoned that these moderate living conditions may have been responsible for the absence of a clear seasonal pattern in densities of the amphipod, an observation also made in this study. It should be emphasised that $U$. poseidonis was regularly found in tube-free sand, which is obviously an acceptable environment for the amphipod. It appears however, that $L$. conchilega improves its living conditions.

This study has shown that even relatively inconspicuous biogenic structures potentially affect the benthic community, albeit more modestly than complex and prominent structures. The presence of single Lanice conchilega enables some species such as scaleworms to colonise an area and the tubes may improve environmental conditions for other benthos dwellers, allowing them to sustain higher population densities.

The example of Urothoe poseidonis shows that the interaction between host and associate may be obscure and can only be understood with detailed knowledge of the autecology of the species involved. In the case of U. poseidonis, this study has shed some light on its ecology, but the exact nature of the relationship between Lanice conchilega and $U$. poseidonis remains speculative.

While dense populations of Lanice conchilega may be important in shaping their habitat, at low densities their measurable impact is limited to the small-scale distribution patterns of the benthic fauna. It certainly is responsible for small-scale variations in the distribution of species, species richness and abundance in Rhossili Bay.

Acknowledgements. This manuscript was greatly improved by comments of 5 anonymous reviewers. Many thanks to P. Hayward for supporting this study. Thanks also to I. Tew and K. Naylor for their help in running the laboratory experiment, and to A. Goffin who carried out the sediment analyses. Many thanks to S. Degraer, F. Glen and D. Callaway for invaluable comments on the first draft of the manuscript.

\section{LITERATURE CITED}

Angel A, Ojeda FP (2001) Structure and trophic organization of subtidal fish assemblages on the northern Chilean coast: the effect of habitat complexity. Mar Ecol Prog Ser 217:81-91

Ban SM, Nelson WG (1987) Role of Diopatra cuprea Bosc (Polychaeta: Onuphidae) tubes in starting a subtropical infaunal community. Bull Mar Sci 40:11-21

Bolam SG, Fernandes TF (2003) Dense aggregations of tube- building polychaetes: response to small-scale disturbances. J Exp Mar Biol Ecol 269:197-222

Bomkamp RE, Page HM, Dugan JE (2004) Role of food subsidies and habitat structure in influencing benthic communities of shell mounds at sites of existing and former offshore oil platforms. Mar Biol 146:201-211

Buhr KJ (1976) Suspension-feeding and assimilation efficiency in Lanice conchilega (Polychaeta). Mar Biol 38: 373-383

Buhr KJ, Winter JE (1977) Distribution and maintenance of a Lanice conchilega association in the Weser estuary (FRG), with special reference to the suspension-feeding behaviour of Lanice conchilega. In: Keegan BF, Ceidigh PO, Boaden PJS (eds) Biology of benthic organisms; 11th European Symposium on Marine Biology. Pergamon Press, Oxford, p 101-113

Callaway R (2003a) Juveniles stick to adults: recruitment of the tube-dwelling polychaete Lanice conchilega (Pallas, 1766). Hydrobiologia 503:121-130

Callaway R (2003b) Long-term effects of imitation polychaete tubes on benthic fauna: they anchor Mytilus edulis (L.) banks. J Exp Mar Biol Ecol 283:115-132

Carey DA (1987) Sedimentological effects and palaeoecological implications of the tube-building polychaete Lanice conchilega (Pallas). Sedimentology 34:49-66

Castilla JC, Lagos NA, Cerda M (2004) Marine ecosystem engineering by the alien ascidian Pyura praeputialis on a mid-intertidal rocky shore. Mar Ecol Prog Ser 268: $119-130$

Clarke KR, Warwick RM (1994) Change in marine communities: an approach to statistical analysis and interpretation. Natural Environment Research Council, Swindon

DeGrave (1999) The influence of sedimentary heterogeneity on within maerl bed differences in infaunal crustacean community. Estuar Coast Shelf Sci 49:153-163

Diaz RJ, Cutter GR, Able KW (2003) The importance of physical and biogenic structure to juvenile fishes on the shallow inner continental shelf. Estuaries 26:12-20

Dubois S, Retiere C, Olivier F (2002) Biodiversity associated with Sabellaria alveolata (Polychaeta: Sabellariidae) reefs: effects of human disturbances. J Mar Biol Assoc UK 82: $817-826$

Eckmann JE, Nowell ARM, Jumars PA (1981) Sediment destabilization by animal tubes. J Mar Res 39:361-374

Edgar GJ (1999) Experimental analysis of structural versus trophic importance of seagrass beds. II. Effects on fishes, decapods and cephalopods. Vie Milieu 49:249-260

Fincham AA (1970) Amphipods in the surf plankton. J Mar Biol Assoc UK 50:177-198

Forster S, Graf G (1995) Impact of irrigation on oxygen flux into the sediment: intermittent pumping by Callianassa subterranea and 'piston-pumping' by Lanice conchilega. Mar Biol 123:335-346

Friedrichs M, Graf G, Springer B (2000) Skimming flow induced over a simulated polychaete tube lawn at low population densities. Mar Ecol Prog Ser 192:219-228

Hartmann-Schröder G (1996) Polychaeta, Vol 58. Gustav Fischer Verlag, Jena

Hertweck G (1995) Verteilung charakteristischer Sedimentkörper und der Benthossiedlungen im Rückseitenwatt der Insel Spiekeroog, südliche Nordsee. 1. Ergebnis der Wattkartierung 1988-92. Senckenb Marit 26:81-94

Holthe T (1978) The zoogeography of the Terebellomorpha (Polychaeta) of the northern European waters. Sarsia 63: 191-198

Khaitov VM, Fokin MV, Nicolaeva AM (1999) Structure of communities associated with dense assemblages of the 
tube-dwelling polychaete Polydora quadrilobata Jacobi (Spionidae) in the White Sea. Hydrobiologia 393:221-226

Kühl H (1963) Über die Nahrung der Scharbe (Limanda limanda L.). Arch Fisch Wiss 14:8-17

Lackschewitz D, Reise K (1998) Macrofauna on flood delta shoals in the Wadden Sea with an underground association between the lugworm Arenicola marina and the amphipod Urothoe poseidonis. Helgol Meeresunters 52: $147-158$

Lawton JH (1994) What do species do in ecosystems? Oikos 71:367-374

Luckenbach MW (1986) Sediment stability around animal tubes: the roles of hydrodynamic processes and biotic activity. Limnol Oceanogr 31:779-787

Luckenbach MW (1987) Effects of adult infauna on new recruits: implications for the role of biogenic refuges. J Exp Mar Biol Ecol 105:197-206

Meyer DL, Townsend EC (2000) Faunal utilization of created intertidal eastern oyster (Crassostrea virginica) reefs in the southeastern United States. Estuaries 23:34-45

Netto SA, Warwick RM, Attrill MJ (1999) Meiobenthic and macrobenthic community structure in carbonate sediments of Rocas Atoll (north-east, Brazil). Estuar Coast Shelf Sci 48:39-50

Peachey RL, Bell SS (1997) The effects of mucous tubes on the distribution, behavior and recruitment of seagrass meiofauna. J Exp Mar Biol Ecol 209:279-291

Petersen B, Exo KM (1999) Predation of waders and gulls on Lanice conchilega tidal flats in the Wadden Sea. Mar Ecol Prog Ser 178:229-240

Phillips TM, Lovell CR (1999) Distributions of total and active bacteria in biofilms lining tubes of the onuphid polychaete Diopatra cuprea. Mar Ecol Prog Ser 183:169-178

Ragnarsson SA, Raffaelli D (1999) Effects of the mussel Mytilus edulis L. on the invertebrate fauna of sediments. J Exp Mar Biol Ecol 241:31-43

Rees EIS, Bergmann M, Galanidi M, Hinz H, Shucksmith R, Kaiser MJ (2005) An enriched Chaetopterus tube mat biotope in the eastern English Channel. J Mar Biol Assoc UK 85:323-326

Editorial responsibility: Otto Kinne (Editor-in-Chief), Oldendorf/Luhe, Germany
Reise K (2002) Sediment mediated species interactions in coastal waters. J Sea Res 48:127-141

Ropert M, Dauvin JC (2000) Renewal and accumulation of a Lanice conchilega (Pallas) population in the baie des Veys, western Bay of Seine. Oceanol Acta 23:529-546

Salvat B (1967) La macrofaune carcinologique endogé des sédiments meubles intertidaux (Tanaidacés, Isopodes et Amphipodes), éthologie, bionomie et cycle biologique. Mem Mus Natl Hist Nat Ser A:1-275

Talman SG, Norkko A, Thrush SF, Hewitt JE (2004) Habitat structure and the survival of juvenile scallops Pecten novaezelandiae: comparing predation in habitats with varying complexity. Mar Ecol Prog Ser 269:197-207

Trueblood DD (1991) Spatial and temporal effects of terebellid polychaete tubes on soft-bottom community structure in Phosphorescent Bay, Puerto Rico. J Exp Mar Biol Ecol 149:139-159

Tsuchiya M, Nishihira M (1986) Islands of Mytilus edulis as a habitat for small intertidal animals: effect of Mytilus age structure on the species composition of the association fauna and community organization. Mar Ecol Prog Ser 31: 171-178

Underwood TJ (1997) Experiments in ecology. Cambridge University Press, Cambridge

Vader W (1978) Association between amphipods and echinoderms. Astarte 11:123-134

Van Gaever S, Vanreusel A, Hughes JA, Bett B, Kiriakoulakis $\mathrm{K}$ (2004) The macro- and micro-scale patchiness of meiobenthos associated with the Darwin Mounds (northeast Atlantic). J Mar Biol Assoc UK 84:547-556

Woodin SA (1978) Refuges, disturbance, and community structure: a marine soft-bottom example. Ecology 59: $274-284$

Ziegelmeier E (1952) Beobachtungen über den Röhrenbau von Lanice conchilega (Pallas) im Experiment und am natürlichen Standort. Helgol Wiss Meeresunters 4: 107-129

Zühlke R (2001) Polychaete tubes create ephemeral community patterns: Lanice conchilega (Pallas, 1766) associations studied over six years. J Sea Res 46:261-272

Submitted: February 28, 2005; Accepted: July 6, 2005

Proofs received from author(s): January 9, 2005 